\title{
Specificity of Phenotypic Adaptation of Bacillus cereus to Tetracycline
}

\author{
By R. H. CONNAMACHER \\ Department of Pharmacology, School of Medicine, University of Pittsburgh, \\ Pittsburgh, Pennsylvania, I5213, U.S.A.
}

(Accepted for publication 7 October 1968)

\begin{abstract}
SUMMARY
Bacillus cereus strain $569 \mathrm{H}$ became resistant to tetracyclines, arsenite and cyanide. The degree of resistance acquired was independent of the inducing concentration up to $2 \times 10^{-5} \mathrm{M}$. Recovery from tetracycline and arsenite inhibition involved a change in the bacteria themselves; cyanide resistance involved also the destruction of this inhibitory agent. Although there were many similarities between the tetracycline and arsenite recoveries, two distinct mechanisms were involved. Adaptation to tetracycline was not observed with B. megaterium, Pseudomonas aeruginosa or Escherichia coli. Bacillus megaterium and $E$. coli did adapt to cyanide. Although polymyxin B was inactive by itself against $B$. cereus, lytic activity due to the antibiotic was seen when a tetracycline was also present in the medium. The lytic activity ceased when the bacilli recovered from tetracycline. Bacillus cereus did not adapt to other inhibitors of protein synthesis, RNA synthesis or oxidative phosphorylation.
\end{abstract}

\section{INTRODUCTION}

A non-genetic adaptation to two different bacteriostatic substances, tetracycline (Connamacher, Mandel \& Hahn, 1966, 1967) and arsenite (Mandel, Mayersak \& Riis, 1965), occurs with Bacillus cereus $569 \mathrm{H}$. In each case, recovery has been due to a decreased intracellular concentration of the inhibitory agent with very little chemical change of the drug. And yet each agent is believed to act by a different mechanism: tetracycline by binding to the $30 \mathrm{~S}$ ribosomes at the point reserved for tRNA (Connamacher \& Mandel, 1968) and arsenite by competition with phosphate in energyproducing mechanisms (Mandel et al. 1965). Because of the similarity in the way the bacteria adapt to the two very different substances, it seemed possible that we were working with a general adaptive mechanism to any toxic agent. Therefore, the present work was done to determine: $(a)$ the spectrum of toxic agents to which the bacteria could adapt; $(b)$ whether there was a relationship between the adaptation due to arsenite and to tetracycline; $(c)$ whether any relationship between arsenite and tetracycline could be seen in a genetically resistant Escherichia coli K-I2. During our work a third agent, cyanide, was found to exhibit the same over-all pattern as did arsenite and tetracycline, and a potentiation was discovered in $B$. cereus between tetracycline and the usually ineffective polymyxin B.

\section{METHODS}

Materials. Tetracycline-HCl was provided by the Lederle and Pfizer Laboratories. Chlortetracycline- $\mathrm{HCl}$ and demethylchlortetracycline- $\mathrm{HCl}$ were supplied by Lederle 
Laboratories, oxytetracycline-HCl by Pfizer Laboratories. The author is grateful to both companies for their assistance.

Bacillus cereus strain $569 \mathrm{H}$ was provided by Dr H. G. Mandel (Department of Pharmacology, The George Washington University School of Medicine). Escherichia coli $\mathrm{K}-\mathrm{I} 2$ strains, one requiring methionine but sensitive to tetracycline and the other requiring thiamine + threonine + leucine and resistant to tetracycline, chloramphenicol, streptomycin and sulphonamides, were kindly furnished by Dr Kazuo Izaki (Department of Agricultural Chemistry, University of Tokyo, now of the Faculty of Agriculture, Tohoku University, Sendai, Japan).

Casein hydrolysate was bought from Difco Laboratories and was the only brand which dissolved completely in the medium of Matthews \& Smith (1956). Other inhibitors and materials came from commercial sources.

Growth of bacteria. Bacillus cereus $569 \mathrm{H}$ and $B$. megaterium were grown in a casein hydrolysate + salts medium (Matthews \& Smith, 1956). Escherichia coli K-I2 was grown in a nutrient broth + glucose medium supplemented with $1 \mathrm{O}^{-5} \mathrm{M}$-thiamine. Antimicrobial agents were added in aqueous solution or suspension in volumes less than $2 \%$ of the total. All bacteria were grown aerobically in an Eberbach waterbath shaker at $37^{\circ}$. Growth was measured turbidimetrically in a Bausch and Lomb Spectronic 20 or a Gilford Model 240 spectrophotometer.

Inter-relationships between inhibitory agents. Many studies consisted of measuring the growth of bacteria in the presence of two compounds added simultaneously or sequentially. In one series of experiments, Bacillus cereus was grown in the presence of either $6 \times 10^{-6} \mathrm{M}$-tetracycline or $\mathrm{I} \cdot 5 \times 10^{-4} \mathrm{M}$-arsenite until recovery. Each sample was then divided into three parts: one received additional $6 \times 10^{-6} \mathrm{M}$-tetracycline, one received $\mathrm{I} \cdot 5 \times 10^{-4} \mathrm{M}$-arsenite and one was kept as control. The growth of each sample was followed as extinction at $540 \mathrm{~m} \mu\left(E_{540}\right)$. In other experiments, after adaptation, the medium was removed from the culture by centrifugation at room temperature and the deposited bacteria washed once with new medium, resuspended in new medium, and the experiment continued as described above. This type of experiment was also done to determine the interrelationships of adaptation between cyanide and tetracycline, and between cyanide and arsenite.

\section{RESULTS}

Adaptation and cross-resistance within the tetracycline family

Bacillus cereus $569 \mathrm{H}$ can become phenotypically resistant to tetracycline, oxytetracycline, chlortetracycline (Fig. I) and demethylchlortetracycline (Fig. 2). The effect of oxytetracycline was the lowest, followed in increasing order by tetracycline, demethylchlortetracycline, chlortetracycline. After bacteria adapted to $6 \times 10^{-6} \mathrm{M}$-tetracycline, they were completely refractory to media containing that amount of drug plus additional $6 \times \mathrm{IO}^{-6} \mathrm{M}$-tetracycline, demethylchlortetracycline or oxytetracycline. Additional chlortetracycline $\left(6 \times \mathrm{IO}^{-6} \mathrm{M}\right)$ was somewhat inhibitory in tetracycline-adapted bacilli, although the same dose without antibiotic pretreatment caused a complete and irreversible arrest of bacterial growth. 


\section{Cross-resistance between antibiotics}

Streptomycin $\left(5 \cdot 2 \times \mathrm{IO}^{-5} \mathrm{M}\right)$, chloramphenicol $\left(3 \times \mathrm{IO}^{-5} \mathrm{M}\right)$, erythromycin $\left(\mathrm{I} \cdot 3 \times 1 \mathrm{O}^{-6}\right.$ M), cyclohexamide $\left(\mathrm{I} \cdot \mathrm{I} \times \mathrm{IO}^{-4} \mathrm{M}\right)$, puromycin $\left(8.5 \times 1 \mathrm{O}^{-5} \mathrm{M}\right)$ and actinomycin $\mathrm{D}$ $\left(1 \mathrm{O}^{-7} \mathrm{M}\right)$ were added separately to bacterial cultures. All but actinomycin $\mathrm{D}$ act by direct inhibition of protein synthesis (Pestka, Marshall \& Nirenberg, 1965; Coutsogeorgopoulos, I966; Wolfe \& Hahn, 1964; Lin, Mosteller \& Hardesty, 1966; Nathans

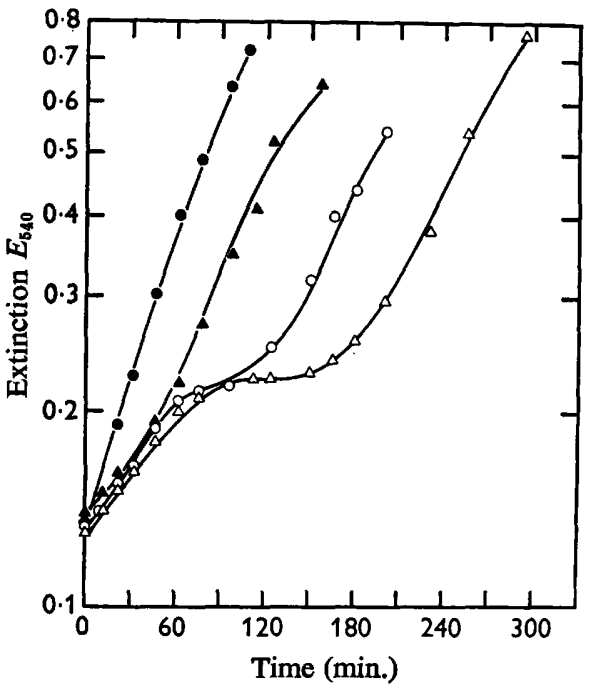

Fig. I

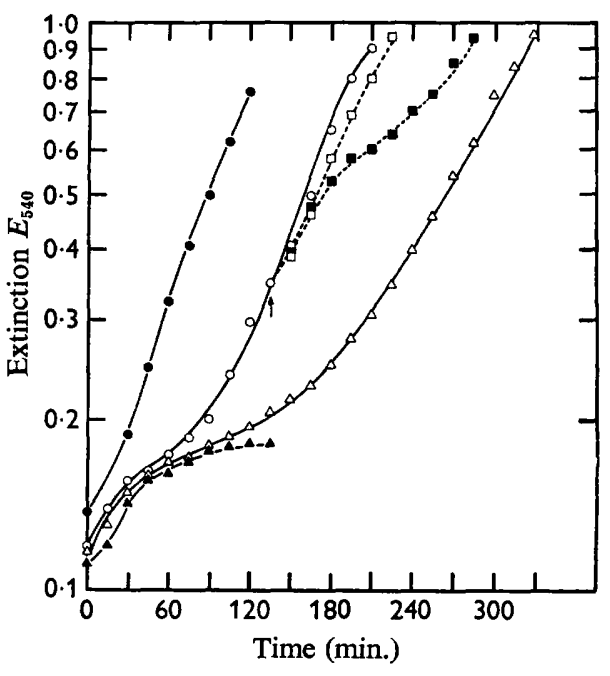

Fig. 2

Fig. I. Adaptation of Bacillus cereus 569H to different tetracyclines. Bacillus cereus was grown in casein hydrolysate + salts medium; growth was measured in a Bausch and Lomb Spectronic 20 spectrophotometer. The extinction at $540 \mathrm{~m} \mu$ was plotted against time. 9 , Control; $\Delta$, oxytetracycline $\left(6 \times 10^{-6} \mathrm{M}\right) ; 0$, tetracycline $\left(6 \times 10^{-6} \mathrm{M}\right) ; \Delta$, chlortetracycline $\left(2 \times 10^{-6} \mathrm{M}\right)$.

Fig. 2. Effect of several tetracyclines before and after adaptation of Bacillus cereus to $6 \times 10^{-6} \mathrm{M}$-tetracycline. Antibiotics were added either at the origin or at arrow $(\uparrow)$. Other details are the same as Fig. I. Control: $O$, tetracycline $\left(6 \times 10^{-6} \mathrm{M}\right) ; \Delta$, demethylchlortetracycline $\left(6 \times 10^{-6} \mathrm{M}\right) ; \Delta$, chlortetracycline $\left(6 \times 10^{-6} \mathrm{M}\right)$. Added at arrow $(\uparrow)$; $\mathbf{v}$, chlortetracycline $\left(6 \times 10^{-6} \mathrm{M}\right) ; \square$, demethylchlortetracycline $\left(6 \times 10^{-6} \mathrm{M}\right)$; oxytetracycline $\left(6 \times 10^{-6} \mathrm{M}\right)$, not shown, was ineffective after bacterial adaptation of tetracycline.

\& Lipmann, 196I; Reich et al. 1962). Although all of the above antibiotics except cyclohexamide were inhibitory to Bacillus cereus adaptation to these agents did not occur. Neither did adaptation of $B$. cereus to tetracycline affect the pattern of inhibition shown by these antibiotics (Fig. 3).

Polymyxin B ( $\left.1 \cdot 6 \times 10^{-5} \mathrm{M}\right)$ by itself was not inhibitory to Bacillus cereus. However, when bacilli were grown also in the presence of $6 \times 10^{-6} \mathrm{M}$-tetracycline, a steady decrease in extinction was seen (Fig. 4). The lysis continued as long as the bacilli remained sensitive to tetracycline. On recovery from tetracycline inhibition, $B$. cereus lost its sensitivity to polymyxin $\mathbf{B}$. 


\section{Cross-resistance between tetracycline and other inhibitors}

Because both tetracycline (Jones \& Morrison, 1962) and arsenite (Mandel et al. 1965) have been shown to interrupt the energy production of the cell, other energy inhibitors and active transport inhibitors were tested for reversible bacteriostatic action. Ouabain $\left(\mathrm{IO}^{-4} \mathrm{M}\right)$ did not inhibit Bacillus cereus, nor was bacterial inhibition observed with up to $10^{-3} \mathrm{M}$-phloridzin, 2,4-dinitrophenol, ethionine, potassium fluoride, amobarbital, or malonic acid. These agents also did not affect the ability of

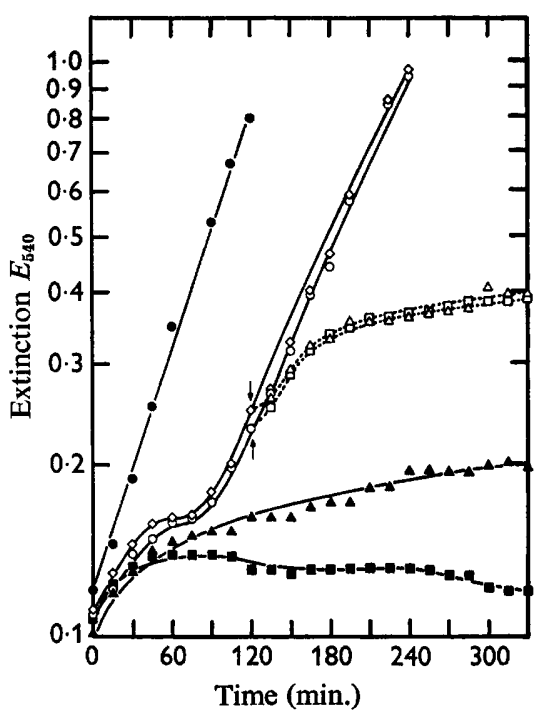

Fig. 3

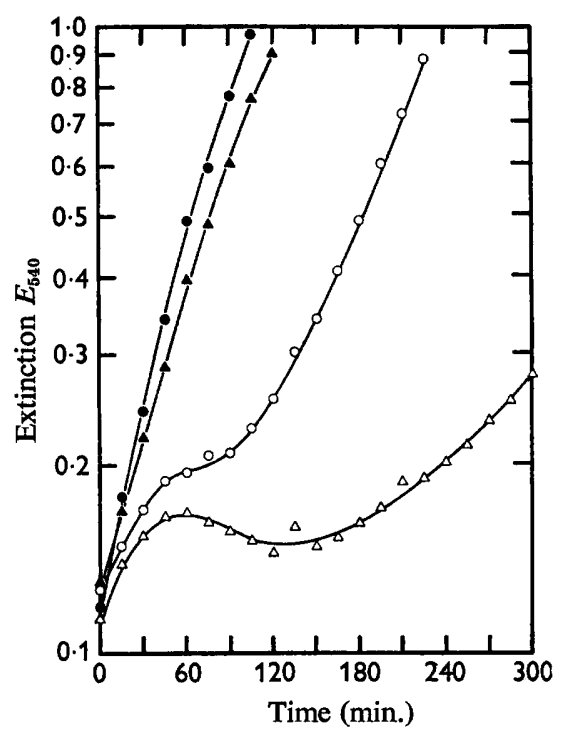

Fig. 4

Fig. 3. Effect of tetracyline adaptation on the action of other antibiotics. Antibiotics erythromycin and puromycin were added to cultures at time $O$ at arrow $(\uparrow) . \bullet, \mathrm{Control;} O$; $\diamond$, tetracycline $\left(6 \times 10^{-6} \mathrm{M}\right) ; \Delta, \triangle$, erythromycin $\left(\mathrm{I} \cdot 3 \times 10^{-6} \mathrm{M}\right) ; \mathbf{\square}, \square$, puromycin $\left(8.5 \times 10^{-6}\right.$ $\mathrm{M})$, closed symbols if added at time $\mathrm{O}$, open symbols if added at arrow. Dashed line signifies a second drug addition.

Fig. 4. Effect of polymyxin B $\left(1 \cdot 6 \times 10^{-5}\right) \mathrm{M}$ on Bacillus cereus $569 \mathrm{H}$ alone or with tetracycline $\left(6 \times 10^{-6} \mathrm{M}\right)$. Experimental details as in Fig. I. $\bullet$, Control; $\Delta$, polymyxin $\mathrm{B}\left(\mathrm{I} \cdot 6 \times 10^{-5}\right.$ $\mathrm{M}) ; \mathrm{O}$, tetracycline $\left(6 \times 10^{-8} \mathrm{M}\right) ; \triangle$, tetracycline $\left(6 \times 10^{-6} \mathrm{M}\right)+$ polymyxin $\mathrm{B}\left(\mathrm{I} \cdot 6 \times 10^{-6} \mathrm{M}\right)$.

B. cereus to adapt to tetracycline. Sodium azide, formaldehyde and iodoacetic acid were inhibitory at concentrations between $10^{-4} \mathrm{M}$ and $10^{-5} \mathrm{M}$, but no recovery was observed. Adaptation of bacteria to tetracycline did not change the inhibitory pattern. Potassium cyanide $\left(\mathrm{IO}^{-3} \mathrm{M}\right)$ caused a period of growth inhibition followed by recovery.

\section{Relationship between tetracycline and arsenite resistance in Bacillus cereus}

Experiments were made to discover whether or not the adaptive mechanism for tetracycline was involved in arsenite adaptation or vice versa. Bacillus cereus was grown in $6 \times 10^{-6} \mathrm{M}$-tetracycline until resumption of full exponential growth. The culture was then divided, $1.5 \times 10^{-4} \mathrm{M}$-arsenite or an additional $6 \times 10^{-6} \mathrm{M}$-tetracycline was added to the tetracycline already present in the medium, and the growth was measured (see Methods). The same experiment was done with bacilli made resistant to 
arsenite. There was a decrease in both cases (Fig. $5 a$ ). To examine the possibility that the attenuation of the bacteriostatic effect was due to a chelation between tetracycline and arsenite in the medium, detoxifying both agents, adapted bacteria were centrifuged at $3000 \mathrm{~g}$ for $10 \mathrm{~min}$., washed once with fresh medium, and resuspended in drug-free medium before the second inhibitory agent was added. These tetracycline-adapted bacilli, when treated in this manner, exhibited an increased sensitivity to arsenite over that seen in non-treated cultures (Fig. $5 b$ ), although adaptation to tetracycline remained. The effect of tetracycline and arsenite after arsenite adaptation remained shortened (Fig. $5 c$ ). This experiment was repeated three times with consistent results.
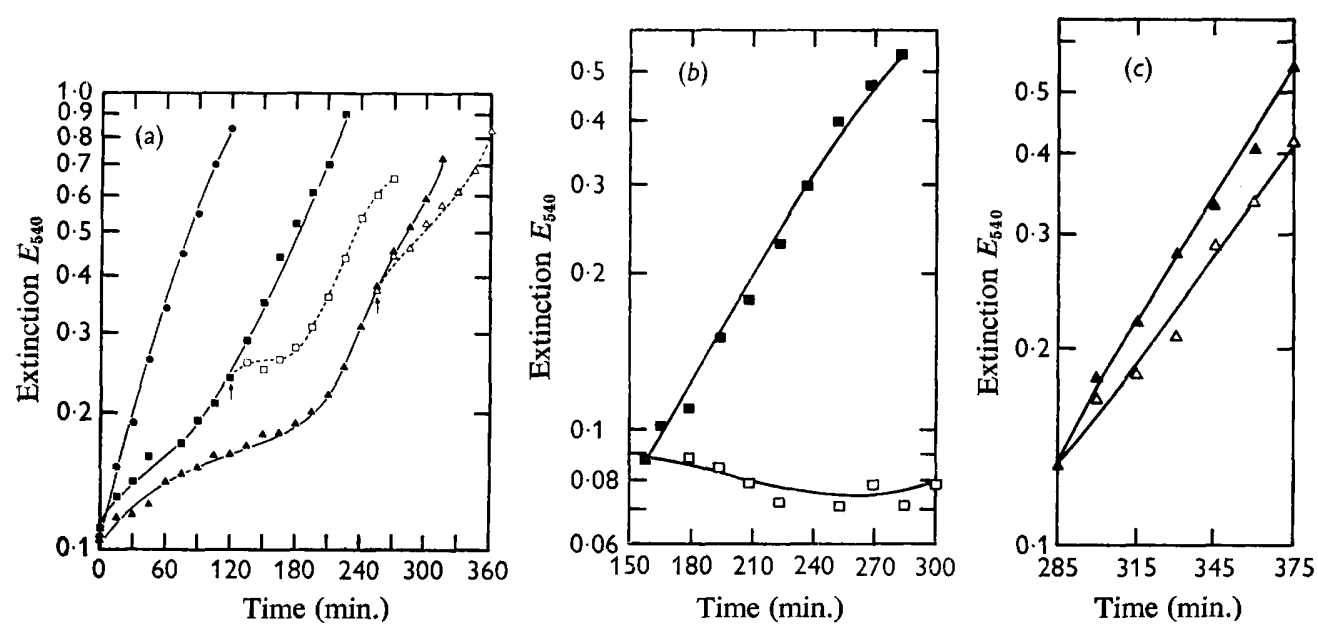

Fig. 5. Interrelationship between tetracycline and arsenite resistances. Bacilli were made resistant to tetracycline or arsenite. (a) Second inhibitory agent added at arrow $(\uparrow)$. (b) Tetracycline removed by centrifuging culture and resuspending bacilli before arsenite addition. (c) Arsenite removed by centrifuging culture and resuspending bacilli before tetracycline addition. Other details are the same as Fig. I. O, Control; $\square$, tetracycline $\left(6 \times 10^{-6} \mathrm{M}\right) ; \square$, arsenite $\left(1.5 \times 10^{-4} \mathrm{M}\right)$ added after tetracycline recovery; $\Delta$, arsenite $\left(\mathrm{I} \cdot 5 \times 10^{-4} \mathrm{M}\right) ; \Delta$, tetracycline $\left(6 \times 10^{-6} \mathrm{M}\right)$ added after arsenite recovery; dashed lines signify the presence of both drugs in the medium.

\section{Relationship between cyanide and arsenite or tetracycline resistances in Bacillus cereus}

Experiments were done to determine the relationship between the cyanide and the arsenite or tetracycline adaptations of Bacillus cereus. The procedure was that used in the previous section. Cyanide recovery did not change the sensitivity to arsenite or tetracycline, nor could it protect the bacilli from a second treatment with cyanide (Fig. 6). Neither tetracycline nor arsenite adaptation changed the sensitivity of $B$. cereus to cyanide. To see whether the bacteria became resistant to cyanide by destroying it, $B$. cereus was grown in the presence or absence of cyanide until adaptation occurred. Both cultures were centrifuged and half of each resuspended in each medium. Cyanideresistant and untreated bacteria were also resuspended in medium containing cyanide that had been incubated in cell-free medium (Fig. 7). The untreated bacteria were not inhibited by medium from cultures of bacilli which had recovered from cyanide inhibition. They were as inhibited by the second introduction of cyanide as they were by the original dose. 


\section{Adaptation of other bacteria to inhibitory agents}

In three other bacterial species, Escherichia coli $\mathrm{K}-\mathrm{I} 2$, Bacillus megaterium and Pseudomonas aeruginosa, recovery from the effects of $6 \times 10^{-6} \mathrm{M}$ tetracycline did not occur within $2.5 \mathrm{hr}$. Recovery from arsenite and cyanide was observed, however, with B. megaterium. Escherichia coli $\mathrm{K}-12$ (sensitive) was more sensitive to tetracycline

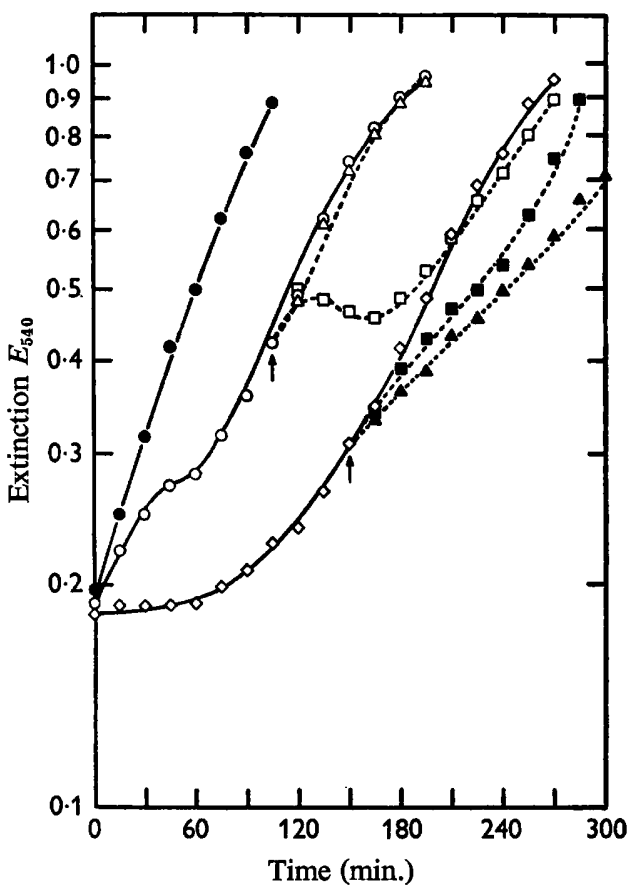

Fig. 6

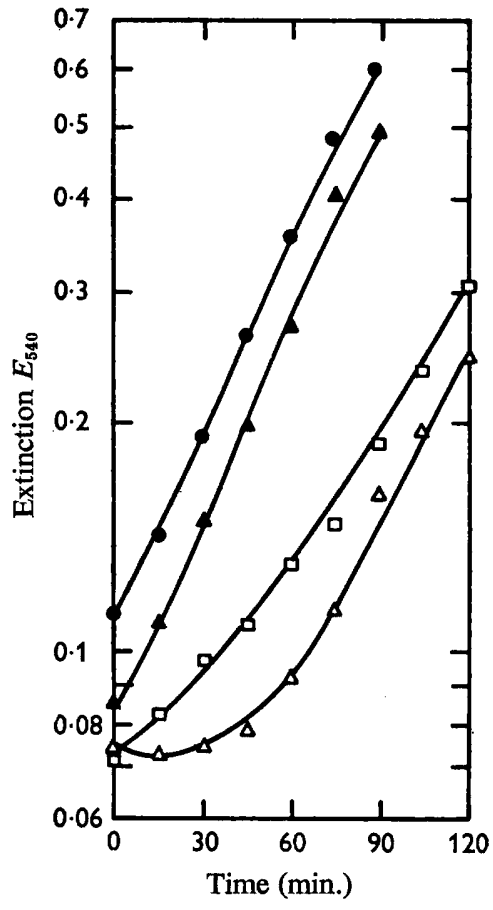

Fig. 7

Fig. 6. Interrelationship between tetracycline and cyanide resistance. Experiment was done in a manner similar to that shown in Fig. $5 a$, except that cyanide $\left(2 \times 10^{-5} \mathrm{M}\right)$ replaced arsenite. Secondary drug additions were made at arrow $(\uparrow)$, and growth curve followed (dashed lines). $\bullet$, Control; $O$, tetracycline $\left(6 \times 10^{-6} \mathrm{M}\right) ; \triangle$, + additional tetracycline to $\left(\mathrm{I} 2 \times 10^{-6} \mathrm{M}\right) ; \square,+\mathrm{KCN}\left(2 \times 10^{-5} \mathrm{M}\right) ; \diamond, \mathrm{KCN}\left(2 \times 10^{-5} \mathrm{M}\right) ; \square+$ tetracycline $\left(6 \times 10^{-6} \mathrm{M}\right) ;$ $\Delta$, tadditional $\mathrm{KCN}\left(2 \times \mathrm{IO}^{-5} \mathrm{M}\right)$.

Fig. 7. Destruction of KCN by Bacillus cereus. B. cereus grown until resistant to KCN $\left(2 \times \mathrm{IO}^{-5} \mathrm{M}\right)$, and medium and bacilli separated. Control bacilli were incubated in that medium, and a sample of medium was incubated with cyanide but without bacilli. KCNadapted bacilli were grown in similar media.

\begin{tabular}{cll} 
Symbol & \multicolumn{1}{c}{ Medium } & \multicolumn{1}{c}{ Bacteria } \\
& Control & Control \\
$\Delta$ & KCN-recovered & Control \\
$\square$ & KCN-bacteria free & KCN-recovered \\
$\triangle$ & KCN-bacteria & Control
\end{tabular}

than was $B$. cereus, being inhibited by concentrations as low as $2 \times 10^{-7} \mathrm{M}$. Unlike $B$. cereus (Connamacher et al. 1967), the rate of growth was proportional to the concentration of tetracycline (Fig. 8). The resistant strain did not respond to $\mathrm{IO}^{-5} \mathrm{M}$ tetracycline. Neither strain of $E$. coli was sensitive to arsenite; both showed a sensitivity to cyanide and an ability to adapt to that drug. 


\section{Relationship between the level of antibiotic resistance and the inducing concentration}

The non-genetic resistance to tetracycline exhibited by Bacillus cereus was not observed with drug concentrations above $5 \times 10^{-5} \mathrm{M}$ (Connamacher et al. 1967). Experiments were done to determine whether the degree of resistance achieved was related to the original 'inducing' concentration of tetracycline. Bacilli were made resistant to concentrations of tetracycline from $2 \times 10^{-6} \mathrm{M}$ to $2 \times 10^{-5} \mathrm{M}$. The degree of resistance achieved was measured by growing the bacilli in additional $2 \times 10^{-5} \mathrm{M}$ to $10^{-4} \mathrm{M}$-tetracycline. The degree of resistance achieved was independent of the 'inducing' concentration (Fig. 9).

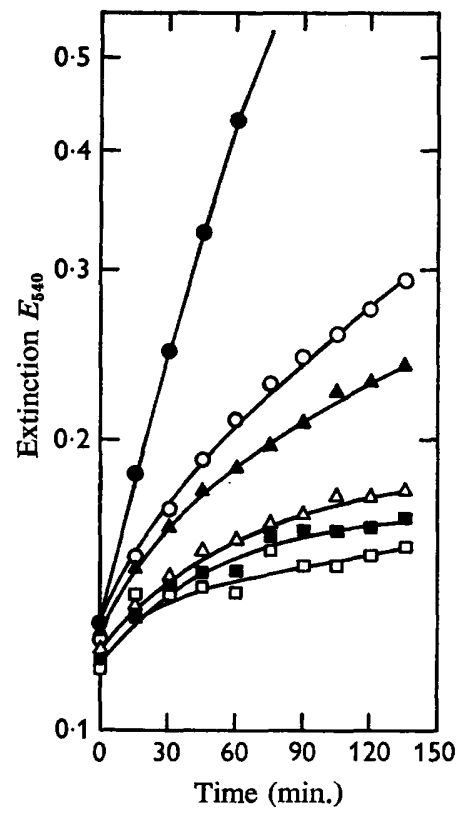

Fig. 8

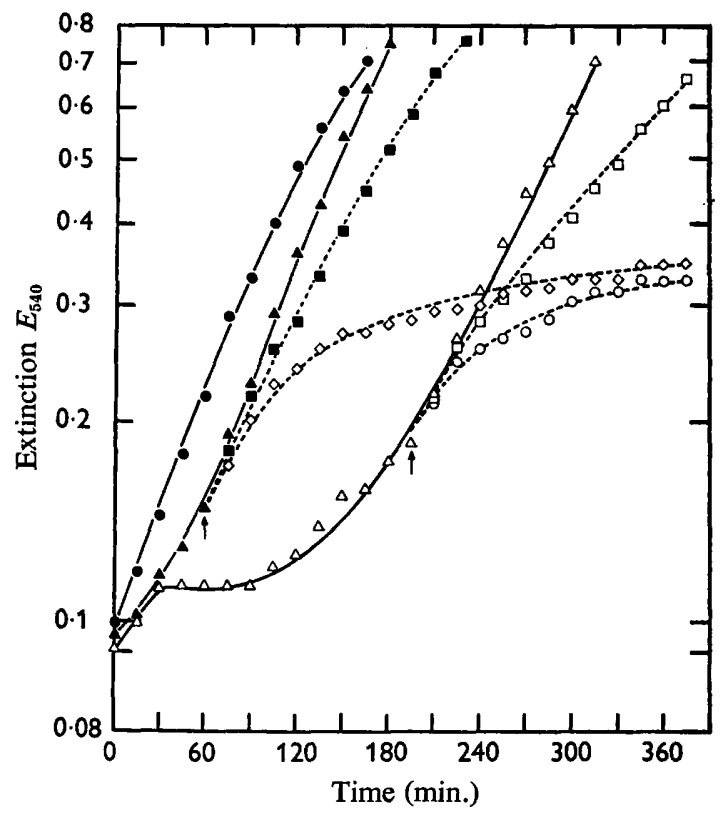

Fig. 9

Fig. 8. Growth of Escherichia coli $\mathrm{K}-12$ (sensitive) in the presence of different concentrations of tetracycline. $E$. coli were grown aerobically in nutrient broth + glucose medium, and turbidity at $540 \mathrm{~m} \mu$ was plotted against time. $\odot$, Control; tetracycline (M): $0,4 \times 10^{-7} ; \Delta, 8 \times 10^{-7}$; $\triangle, 2 \times 10^{-6} ; 0,4 \times 10^{-6} ; \square, 10^{-5}$.

Fig. 9. Action of higher concentrations of tetracycline on Bacillus cereus adapted to $6 \times 10^{-6} \mathrm{M}$ or $\mathrm{I} \cdot 8 \times 10^{-5} \mathrm{M}$ tetracycline. Additional drug added at arrow $(\uparrow)$, and growth curves followed (dashed lines). $\odot$, Control; $\boldsymbol{\Delta}$, tetracycline $\left(6 \times 10^{-6} \mathrm{M}\right) ; \boldsymbol{\square}$, + additional tetracycline $\left(\mathrm{IO}^{-5} \mathrm{M}\right) ; \triangle$, additional tetracycline $\left(5 \times 10^{-5} \mathrm{M}\right) ; \triangle$, tetracycline $\left(\mathrm{I} \cdot 8 \times 1 \mathrm{IO}^{-5} \mathrm{M}\right)$ $\square$, + additional tetracycline $\left(10^{-5} \mathrm{M}\right) ; \mathrm{O}$, +additional tetracycline $\left(5 \times 10^{-5} \mathrm{M}\right)$.

\section{DISCUSSION}

Two aspects of non-genetic resistance to tetracycline are at present under investigation. They are: $(a)$ what are the biochemical events that occur during the adaptation of Bacillus cereus to tetracycline; $(b)$ do they occur in bacilli made resistant through genetic mutation? Because protein, and therefore enzyme, synthesis is totally inhibited 
by $6 \times 10^{-6} \mathrm{M}$-tetracycline (Connamacher \& Mandel, I964; Hash, Wishnick \& Miller, I964), and because energy is required for the adaptation to take place (Connamacher et al. 1966), active mechanisms other than protein synthesis must be responsible. The specificity of the adaptation might give a clue as to the biochemical mechanisms involved.

\section{Interrelationships between the mechanisms of resistance and the inhibitory agent}

The similarity between the adaptations to tetracycline and to arsenite suggested the possibility that both use the same mechanism of adaptation. In both cases, the resistant bacteria contained less drug than did the sensitive ones (Connamacher et al. 1967; Mandel et al. 1965). In the author's experience, the recovery from both compounds must occur within $3 \mathrm{hr}$ or not at all. And both tetracycline- and arseniteadapted bacilli remained susceptible to higher concentrations of the inhibitory agents. Further evidence for the interrelationship between tetracycline and arsenite was seen in the decreased sensitivity to arsenite after tetracycline adaptation in the absence of washing. It was difficult to evaluate the apparent decrease in tetracycline sensitivity after bacilli became resistant to arsenite since it was possible that tetracycline chelated with arsenite in the medium, inactivating both (the arsenite concentration was 25 times that of tetracycline, so that even washing might not remove enough of the trivalent anion). Despite this apparent relationship, it now appears that each resistance has its own mechanism of onset. When the medium from tetracyclineadapted bacilli was exchanged for drug-free medium before addition of arsenite (Fig. 5), the bacilli remained resistant to tetracycline. However, they became more sensitive to arsenite than they were before adaptation to tetracycline. Secondly, the related organism, Bacillus megaterium, remained sensitive to tetracycline even when it had adapted to arsenite. Lastly, if this adaptation was a general mechanism, it would be expected to be seen in many instances. Actually, phenotypic resistance could only be demonstrated with tetracycline, arsenite and cyanide. The recovery of bacilli from cyanide inhibition appeared to use a mechanism different from either of the two described above. Firstly, the recovery occurred in all bacilli studies, whether or not tetracycline or arsenite adaptation also took place. Secondly, bacilli destroyed the cyanide in the medium, which was not the case with the other two drugs.

\section{Independence of adaptive tetracycline concentration and degree of resistance achieved}

With most non-genetic adaptations there is a relationship between the amount of a drug used to promote resistance and the degree of resistance achieved. With tetracycline this relationship was not observed. Whatever the concentration of tetracycline used, from the slightly inhibitory $2 \times 10^{-6} \mathrm{M}$ to $2 \times 10^{-5} \mathrm{M}$, above which the bacilli did not recover, the degree of resistance remained the same. This would suggest that tetracycline was acting as a derepressor, except that protein synthesis was completely inhibited. A more likely possibility suggested by Connamacher et al. (1967) is that two mechanisms are involved in tetracycline entry, one active and one passive. All drug concentrations used completely inhibited the active phase. However, high extracellular concentrations of tetracycline still caused sufficient entry of the antibiotic by passive diffusion to inhibit the bacterial growth. 


\section{Interrelationship between tetracycline and polymyxin $B$}

Polymyxin B is normally effective against only those Gram-positive bacteria which are highly sensitive to lysozyme (Newton, 1956). Bacillus cereus is not such an organism, and it is not normally susceptible to polymyxin B. In the presence of tetracycline, however, a bactericidal effect of polymyxin B was seen. Once tetracycline adaptation occurred, polymyxin action ceased. Tetracycline thus appeared to alter the bacilli in such a way as to allow polymyxin B access to the cell membrane of Gram-positive bacteria. On recovery of the bacilli from the effects of tetracycline, this access was lost. Work on this aspect is continuing.

This work was supported by Grant no. AI-08192-0I from the United States National Institute of Allergy and Infectious Diseases. The author thanks Miss Marlease Williams for careful technical assistance and Dr W. S. Vincent for aid in the preparation of this manuscript. He also thanks the Lederle Laboratories and Pfizer Laboratories for gifts of the tetracyclines used in this paper.

\section{REFERENCES}

ConNAmacher, R. H. \& MANDEL, H. H. (1964). Studies on the mechanism of action of tetracycline on B. cereus. Fedn. Proc. 23, 388.

CONNAMACHER, R. H. \& MANDEL, H. G. (1968). Intracellular localization of tetracycline in bacteria. Biochim. biophys. Acta 166, 475.

Connamacher, R. H., Mandel, H. G. \& Hahn, F. E. (1966). Non-genetic resistance by Bacillus cereus to tetracycline. Fedn. Proc. Fedn. Am. Socs exp. Biol. 25, 344.

ConNAMACHER, R. H., MANDEL, H. G. \& HAHN, F. E. (I967). Adaptation of populations of Bacillus cereus to tetracycline. Molec. Pharmac. 3, 586.

Coutsogeorgopoulos, C. (1966). On the mechanism of action of chloramphenicol in protein synsynthesis. Biochim. biophys. Acta 129, 214.

HASH, J. H., WishincK, M. \& MilleR, P. A. (1964). On the mechanism of action of the tetracycline antibiotics in Staphylococcus aureus. J. biol. Chem. 239, 2070.

Jones, J.G. \& Morrison, G. A. (1962). The bacteriostatic actions of tetracycline and oxytetracycline. J. Pharm. Pharmac. 14, 808.

LiN, S. Y., MostelleR, R. D., \& HARDESTY, B. (1966). The mechanism of sodium fluoride and cyclohexamide inhibition of haemoglobin biosynthesis in the cell-free reticulocyte system. $J$. molec. Biol. 21, 5 I.

MANDel, H. G., MAYersak, J.S. \& RiIs, M. (1965). The action of arsenic on Bacillus cereus. J. Pharm. Pharmac. 17, 794.

MatTHEWs, R. E. F. \& SMITH, J. D. (1956). Distribution of 8-Azaguanine in the nucleic acids of Bacillus cereus. Nature, Lond. 177, $27 \mathrm{I}$.

NATHANS, D. L \& LipMANN, F. (I96I). Amino acid transfer from amino acylribonucleic acids to protein on ribosomes of Escherichia coli. Proc. natn. Acad. Sci. U.S.A., 47, 497.

Newton, B. A. (1956). The properties and mode of action of polymyxin. Bact. Rev. 20, I4.

PestKa, S., Marshall, R. \& Nirenberg, M. (1965). RNA codewords and protein synthesis. V. Effect of streptomycin on the formation of ribosome-sRNA complexes. Proc. natn. Acad. Sci., U.S.A. $53,639$.

Reich, E., Frankin, R. M., Shatkin, A. J. \& Tatum, E. L. (1962). Action of actinomycin D on animal cells and viruses. Proc. natn. Acad. Sci. U.S.A. 48, 1238.

WolfE, A. D. \& HAHN, F. E. (1964). Erythromycin: mode of action. Science, N.Y. 143, 1445. 